Acta Crystallographica Section E

Structure Reports

Online

ISSN 1600-5368

\section{Thomas C. Lewis and Derek A. Tocher*}

Department of Chemistry, University College London, 20 Gordon Street, London WC1H OAJ England

Correspondence e-mail: d.a.tocher@ucl.ac.uk

\section{Key indicators}

Single-crystal X-ray study

$T=150 \mathrm{~K}$

Mean $\sigma(\mathrm{C}-\mathrm{C})=0.003 \AA$

$R$ factor $=0.043$

$w R$ factor $=0.102$

Data-to-parameter ratio $=11.5$

For details of how these key indicators were automatically derived from the article, see http://journals.iucr.org/e.

\title{
5,5-Dihydroxybarbituric acid monohydrate (alloxan dihydrate)
}

The title compound, $\mathrm{C}_{4} \mathrm{H}_{4} \mathrm{~N}_{2} \mathrm{O}_{5} \cdot \mathrm{H}_{2} \mathrm{O}$, was crystallized from both tetrahydrofuran and 1,4-dioxane solutions of alloxan as part of an experimental polymorph screen on alloxan.

\section{Comment}

It has previously been reported that alloxan has two hydrates, viz. 5,5-dihydroxybarbituric acid (Singh, 1965; Harrowfield et al., 1989) and 5,5-dihydroxybarbituric acid trihydrate (Mootz \& Jeffrey, 1965). The crystal structure of a new hydrate of alloxan, namely 5,5-dihydroxybarbituric acid monohydrate, (I), has one organic molecule and one water molecule in the asymmetric unit (Fig. 1). The heterocyclic ring has an envelope conformation with the flap at $\mathrm{C} 5$, with the angle between the mean $\mathrm{C} 4 / \mathrm{N} 3 / \mathrm{C} 2 / \mathrm{N} 1 / \mathrm{C} 6$ and $\mathrm{C} 4 / \mathrm{C} 5 / \mathrm{C} 6$ planes being $20.1(2)^{\circ}$. The $\mathrm{C}-\mathrm{N}$ bond lengths are in the range 1.360 (2)-1.378 (2) $\AA$, with the bond lengths associated with the $s p^{3}$-hybridized carbon being 1.536 (2) and 1.527 (2) $\AA$ for $\mathrm{C} 4-\mathrm{C} 5$ and $\mathrm{C} 5-$ C6, respectively.<smiles>O=C1NC(=O)C(O)(O)C(=O)N1</smiles>

(I)
Received 25 August 2004 Accepted 1 September 2004 Online 11 September 2004
The crystal packing (Fig. 2) consists of a series of ribbon motifs arranged in an overall sheet structure. Water molecules lie in the sheets and between the ribbons. Each water molecule acts as a hydrogen-bond donor to a carbonyl group in the same sheet and to a hydroxyl group on a molecule in the adjacent sheet. Each water molecule also acts as a hydrogenbond acceptor for a hydroxyl group on a molecule in the same sheet. The axial hydroxyl group on each molecule acts as a hydrogen-bond donor to the unique carbonyl of a molecule in an adjacent sheet. The $D \cdots A$ distances within the sheets are in the range $2.6380(19)-2.9516(19) \AA$, whilst the distances between the sheets are 2.6958 (17) and 2.9973 (19) $\AA$. All potential hydrogen-bond acceptors and donors participate in the hydrogen bonding.

\section{Experimental}

5,5-Dihydroxybarbituric acid monohydrate was crystallized over a number of weeks by slow evaporation of tetrahydrofuran and 1,4dioxane solutions of alloxan $\left(0.002-0.03 \mathrm{~mol} \mathrm{dm}^{-3}\right)$ at room temperature, forming colourless plate crystals. 
Crystal data

$$
\begin{aligned}
& \mathrm{C}_{4} \mathrm{H}_{4} \mathrm{~N}_{2} \mathrm{O}_{5} \cdot \mathrm{H}_{2} \mathrm{O} \\
& M_{r}=178.11 \\
& \text { Triclinic, } P \overline{1} \\
& a=6.6730(11) \AA \\
& b=7.5834(13) \AA \\
& c=7.6157(13) \AA \\
& \alpha=105.401(3)^{\circ} \\
& \beta=93.134(3)^{\circ} \\
& \gamma=115.089(2)^{\circ} \\
& V=330.26(10) \AA^{3}
\end{aligned}
$$

$Z=2$

$D_{x}=1.791 \mathrm{Mg} \mathrm{m}^{-3}$

Mo $K \alpha$ radiation

Cell parameters from 712

reflections

$\theta=2.8-25.0^{\circ}$

$\mu=0.17 \mathrm{~mm}^{-1}$

$T=150$ (2) K

Plate, colourless

$0.23 \times 0.11 \times 0.07 \mathrm{~mm}$

\section{Data collection}

Bruker SMART APEX

diffractometer

Narrow-frame $\omega$ scans

Absorption correction: multi-scan (SADABS; Sheldrick, 1996)

$T_{\min }=0.962, T_{\max }=0.988$

2972 measured reflections

\section{Refinement}

Refinement on $F^{2}$

$R\left[F^{2}>2 \sigma\left(F^{2}\right)\right]=0.043$

$w R\left(F^{2}\right)=0.102$

$S=1.07$

1536 reflections

133 parameters

All $\mathrm{H}$-atom parameters refined

1536 independent reflections 1274 reflections with $I>2 \sigma(I)$

$R_{\text {int }}=0.021$

$\theta_{\max }=28.3^{\circ}$

$h=-8 \rightarrow 8$

$k=-9 \rightarrow 9$

$l=-10 \rightarrow 10$

$$
\begin{gathered}
w=1 /\left[\sigma^{2}\left(F_{o}{ }^{2}\right)+(0.0502 P)^{2}\right. \\
+0.0119 P] \\
\text { where } P=\left(F_{o}^{2}+2 F_{c}^{2}\right) / 3 \\
(\Delta / \sigma)_{\max }<0.001 \\
\Delta \rho_{\max }=0.34 \mathrm{e}^{-3} \\
\Delta \rho_{\min }=-0.24 \AA^{-3}
\end{gathered}
$$

Table 1

Hydrogen-bonding geometry $\left(\AA,^{\circ}\right)$.

\begin{tabular}{lllll}
\hline$D-\mathrm{H} \cdots A$ & $D-\mathrm{H}$ & $\mathrm{H} \cdots A$ & $D \cdots A$ & $D-\mathrm{H} \cdots A$ \\
\hline $\mathrm{N} 1-\mathrm{H} 1 \cdots \mathrm{O}^{\mathrm{i}}$ & $0.89(2)$ & $1.95(2)$ & $2.8366(18)$ & $170.7(19)$ \\
$\mathrm{N} 3-\mathrm{H} 3 \cdots \mathrm{O}^{\mathrm{ii}}$ & $0.81(2)$ & $2.11(2)$ & $2.8736(18)$ & $157(2)$ \\
$\mathrm{O} 7-\mathrm{H} 7 \cdots \mathrm{O} 2{ }^{\mathrm{iii}}$ & $0.87(2)$ & $1.83(3)$ & $2.6958(17)$ & $173(2)$ \\
$\mathrm{O} 8-\mathrm{H} 8 \cdots \mathrm{O} 1 W^{\mathrm{iii}}$ & $0.80(2)$ & $1.87(2)$ & $2.6380(19)$ & $161(2)$ \\
$\mathrm{O} 1 W-\mathrm{H} 1 W \cdots \mathrm{O}^{\mathrm{iv}}$ & $0.92(3)$ & $2.04(3)$ & $2.9516(19)$ & $173(2)$ \\
$\mathrm{O} 1 W-\mathrm{H} 2 W \cdots 7^{\mathrm{ii}}$ & $0.82(3)$ & $2.28(3)$ & $2.9973(19)$ & $147(3)$ \\
\hline
\end{tabular}

Symmetry codes: (i) $1-x,-y, 2-z$; (ii) $1-x, 1-y, 1-z$; (iii) $1+x, y, z$; (iv) $1-x,-y, 1-z$

$\mathrm{H}$ atoms were refined freely with an isotropic model.

Data collection: SMART (Bruker, 2000); cell refinement: SAINT (Bruker, 2000); data reduction: SAINT; program(s) used to solve structure: SHELXS97 (Sheldrick, 1990); program(s) used to refine structure: SHELXL97 (Sheldrick, 1997); molecular graphics: SHELXTL (Bruker, 2000; Bruno et al., 2002); software used to prepare material for publication: SHELXL97.

This research was supported by the EPSRC in funding a studentship for TCL. The authors acknowledge the Research Councils UK Basic Technology Programme for supporting 'Control and Prediction of the Organic Solid State'. For
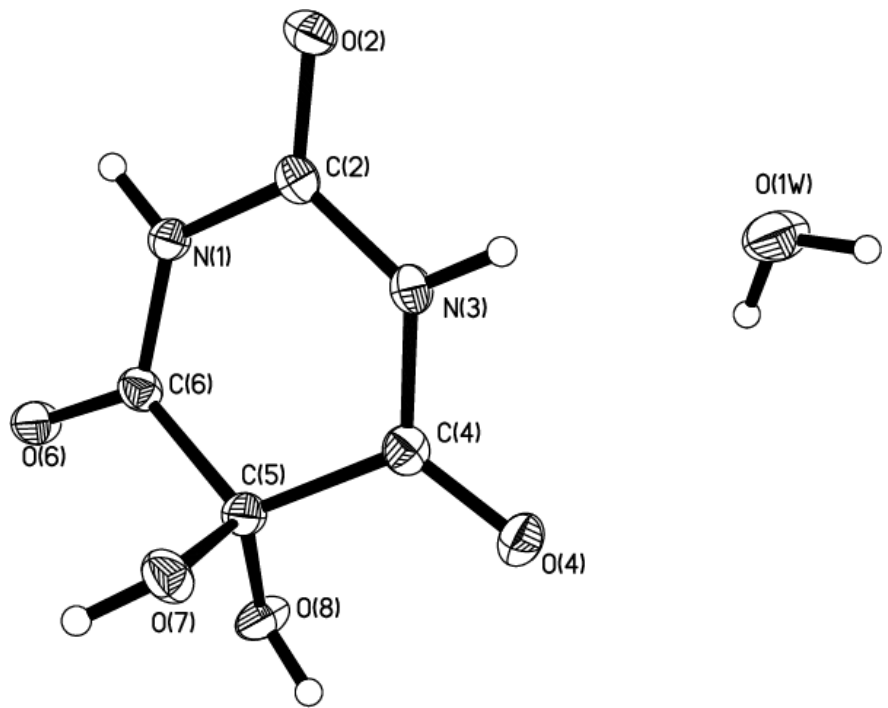

Figure 1

The asymmetric unit of (I), showing the atom-labelling scheme. Displacement ellipsoids are drawn at the $50 \%$ probability level.

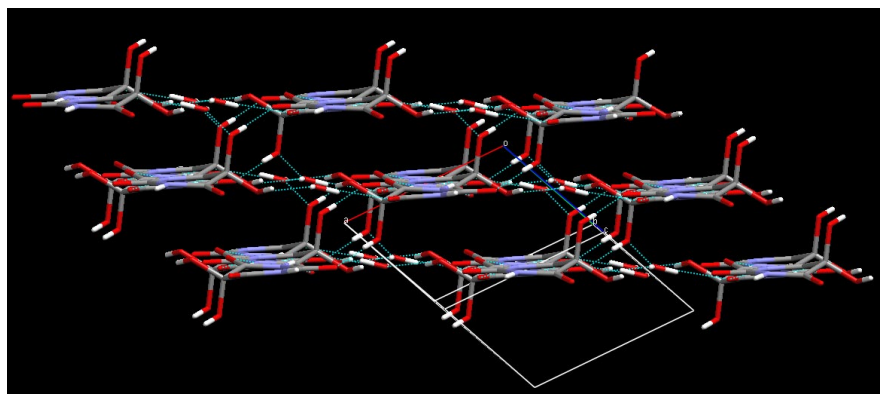

Figure 2

The crystal packing of (I), showing the $\mathrm{N}-\mathrm{H} \cdots \mathrm{O}$ and $\mathrm{O}-\mathrm{H} \cdots \mathrm{O}$ hydrogen-bonding interactions as dashed lines; the view is approximately on to the $(01 \overline{1})$ plane.

more information on this work, please visit http:// www.chem.ucl.ac.uk/basictechorg/.

\section{References}

Bruker (2000). SMART, SAINT and SHELXTL. Bruker AXS Inc., Madison, Wisconsin, USA.

Bruno, Z. J., Cole, J. C., Edgington, P. R., Kessler, M. K., Macrae, C. F., McCabe, P., Pearson, J. \& Taylor, R. (2002). Acta Cryst. B58, 389-397.

Harrowfield, J. M., Skelton, B. W., Soudi, A. A. \& White, A. H. (1989). Aust. J. Chem. 42, 1795-1798.

Mootz, D. \& Jeffrey, G. A. (1965). Acta Cryst. 19, 717-725.

Sheldrick, G. M. (1996). SADABS. University of Göttingen, Germany.

Sheldrick, G. M. (1990). Acta Cryst. A46, 467-473.

Sheldrick, G. M. (1997). SHELXL97. University of Göttingen, Germany.

Singh, C. (1965). Acta Cryst. 19, 759-767. 


\title{
supporting information
}

Acta Cryst. (2004). E60, o1689-o1690 [https://doi.org/10.1107/S1600536804021555]

\section{5,5-Dihydroxybarbituric acid monohydrate (alloxan dihydrate)}

\author{
Thomas C. Lewis and Derek A. Tocher
}

\section{5,5-dihydroxybarbituric acid monohydrate}

\section{Crystal data}

$\mathrm{C}_{4} \mathrm{H}_{4} \mathrm{~N}_{2} \mathrm{O}_{5} \cdot \mathrm{H}_{2} \mathrm{O}$

$M_{r}=178.11$

Triclinic, $P \overline{1}$

$a=6.6730(11) \AA$

$b=7.5834(13) \AA$

$c=7.6157(13) \AA$

$\alpha=105.401(3)^{\circ}$

$\beta=93.134(3)^{\circ}$

$\gamma=115.089(2)^{\circ}$

$V=330.26(10) \AA^{3}$

Data collection

Bruker SMART APEX

diffractometer

Radiation source: fine-focus sealed tube

Graphite monochromator

$\omega$ rotation scans with narrow frames

Absorption correction: multi-scan

(SADABS; Sheldrick, 1996)

$T_{\min }=0.962, T_{\max }=0.988$

\section{Refinement}

Refinement on $F^{2}$

Least-squares matrix: full

$R\left[F^{2}>2 \sigma\left(F^{2}\right)\right]=0.043$

$w R\left(F^{2}\right)=0.102$

$S=1.07$

1536 reflections

133 parameters

0 restraints

Primary atom site location: structure-invariant direct methods
$Z=2$

$F(000)=184$

$D_{\mathrm{x}}=1.791 \mathrm{Mg} \mathrm{m}^{-3}$

Mo $K \alpha$ radiation, $\lambda=0.71073 \AA$

Cell parameters from 712 reflections

$\theta=2.8-25.0^{\circ}$

$\mu=0.17 \mathrm{~mm}^{-1}$

$T=150 \mathrm{~K}$

Plate, colourless

$0.23 \times 0.11 \times 0.07 \mathrm{~mm}$

2972 measured reflections

1536 independent reflections

1274 reflections with $I>2 \sigma(I)$

$R_{\text {int }}=0.021$

$\theta_{\text {max }}=28.3^{\circ}, \theta_{\text {min }}=2.8^{\circ}$

$h=-8 \rightarrow 8$

$k=-9 \rightarrow 9$

$l=-10 \rightarrow 10$

Secondary atom site location: difference Fourier map

Hydrogen site location: inferred from neighbouring sites

All H-atom parameters refined

$w=1 /\left[\sigma^{2}\left(F_{\mathrm{o}}^{2}\right)+(0.0502 P)^{2}+0.0119 P\right]$ where $P=\left(F_{\mathrm{o}}^{2}+2 F_{\mathrm{c}}^{2}\right) / 3$

$(\Delta / \sigma)_{\max }<0.001$

$\Delta \rho_{\max }=0.34$ e $\AA^{-3}$

$\Delta \rho_{\min }=-0.24$ e $\AA^{-3}$

Special details

Geometry. All e.s.d.'s (except the e.s.d. in the dihedral angle between two 1.s. planes) are estimated using the full covariance matrix. The cell e.s.d.'s are taken into account individually in the estimation of e.s.d.'s in distances, angles and torsion angles; correlations between e.s.d.'s in cell parameters are only used when they are defined by crystal symmetry. An approximate (isotropic) treatment of cell e.s.d.'s is used for estimating e.s.d.'s involving 1.s. planes. 
Refinement. Refinement of $F^{2}$ against ALL reflections. The weighted $R$-factor $w R$ and goodness of fit $S$ are based on $F^{2}$, conventional $R$-factors $R$ are based on $F$, with $F$ set to zero for negative $F^{2}$. The threshold expression of $F^{2}>\sigma\left(F^{2}\right)$ is used only for calculating $R$-factors(gt) etc. and is not relevant to the choice of reflections for refinement. $R$-factors based on $F^{2}$ are statistically about twice as large as those based on $F$, and $R$ - factors based on ALL data will be even larger.

Fractional atomic coordinates and isotropic or equivalent isotropic displacement parameters $\left(\hat{A}^{2}\right)$

\begin{tabular}{lllll}
\hline & $x$ & $y$ & $z$ & $U_{\text {iso }} * / U_{\text {eq }}$ \\
\hline O2 & $0.2526(2)$ & $0.32902(18)$ & $0.90727(17)$ & $0.0200(3)$ \\
O4 & $0.6356(2)$ & $0.34785(19)$ & $0.43384(16)$ & $0.0214(3)$ \\
O6 & $0.7119(2)$ & $0.02976(19)$ & $0.86645(17)$ & $0.0207(3)$ \\
O7 & $0.9445(2)$ & $0.42247(18)$ & $0.76705(17)$ & $0.0194(3)$ \\
O8 & $0.7906(2)$ & $0.09757(19)$ & $0.54162(17)$ & $0.0204(3)$ \\
O1W & $0.0411(3)$ & $0.1981(2)$ & $0.2940(2)$ & $0.0339(4)$ \\
N1 & $0.4853(2)$ & $0.1829(2)$ & $0.8839(2)$ & $0.0160(3)$ \\
N3 & $0.4626(2)$ & $0.3615(2)$ & $0.6818(2)$ & $0.0171(3)$ \\
C2 & $0.3919(3)$ & $0.2946(2)$ & $0.8279(2)$ & $0.0156(4)$ \\
C4 & $0.6073(3)$ & $0.3162(2)$ & $0.5801(2)$ & $0.0160(4)$ \\
C5 & $0.7514(3)$ & $0.2387(2)$ & $0.6700(2)$ & $0.0155(4)$ \\
C6 & $0.6456(3)$ & $0.1366(2)$ & $0.8121(2)$ & $0.0154(3)$ \\
H1 & $0.421(4)$ & $0.128(3)$ & $0.969(3)$ & $0.033(6)^{*}$ \\
H3 & $0.398(4)$ & $0.416(3)$ & $0.641(3)$ & $0.035(6)^{*}$ \\
H7 & $1.035(4)$ & $0.383(4)$ & $0.813(3)$ & $0.046(7)^{*}$ \\
H8 & $0.859(4)$ & $0.149(3)$ & $0.472(3)$ & $0.034(6)^{*}$ \\
H1W & $0.107(5)$ & $0.117(4)$ & $0.244(4)$ & $0.059(8)^{*}$ \\
H2W & $0.030(5)$ & $0.268(4)$ & $0.232(4)$ & $0.072(10)^{*}$ \\
& & & & \\
\hline
\end{tabular}

Atomic displacement parameters $\left(\AA^{2}\right)$

\begin{tabular}{lllllll}
\hline & $U^{11}$ & $U^{22}$ & $U^{33}$ & $U^{12}$ & $U^{13}$ & $U^{23}$ \\
\hline O2 & $0.0185(6)$ & $0.0251(7)$ & $0.0231(7)$ & $0.0143(5)$ & $0.0074(5)$ & $0.0101(5)$ \\
O4 & $0.0264(7)$ & $0.0273(7)$ & $0.0190(7)$ & $0.0157(6)$ & $0.0081(5)$ & $0.0136(5)$ \\
O6 & $0.0236(7)$ & $0.0270(7)$ & $0.0256(7)$ & $0.0179(6)$ & $0.0120(5)$ & $0.0175(5)$ \\
O7 & $0.0158(6)$ & $0.0205(7)$ & $0.0262(7)$ & $0.0093(5)$ & $0.0038(5)$ & $0.0121(5)$ \\
O8 & $0.0292(7)$ & $0.0213(7)$ & $0.0204(7)$ & $0.0161(6)$ & $0.0143(5)$ & $0.0116(5)$ \\
O1W & $0.0465(9)$ & $0.0343(8)$ & $0.0412(9)$ & $0.0277(8)$ & $0.0276(8)$ & $0.0235(7)$ \\
N1 & $0.0171(7)$ & $0.0204(7)$ & $0.0173(7)$ & $0.0110(6)$ & $0.0068(6)$ & $0.0116(6)$ \\
N3 & $0.0198(7)$ & $0.0201(7)$ & $0.0191(7)$ & $0.0133(6)$ & $0.0049(6)$ & $0.0106(6)$ \\
C2 & $0.0155(8)$ & $0.0151(8)$ & $0.0173(8)$ & $0.0079(7)$ & $0.0019(6)$ & $0.0056(7)$ \\
C4 & $0.0171(8)$ & $0.0128(8)$ & $0.0192(8)$ & $0.0068(7)$ & $0.0033(6)$ & $0.0068(6)$ \\
C5 & $0.0162(8)$ & $0.0170(8)$ & $0.0172(8)$ & $0.0090(7)$ & $0.0054(6)$ & $0.0084(7)$ \\
C6 & $0.0144(8)$ & $0.0155(8)$ & $0.0186(9)$ & $0.0075(7)$ & $0.0038(6)$ & $0.0077(7)$ \\
\hline
\end{tabular}

Geometric parameters $\left(\AA,^{\circ}\right)$

\begin{tabular}{llll}
\hline $\mathrm{O} 2-\mathrm{C} 2$ & $1.2171(19)$ & $\mathrm{N} 1-\mathrm{C} 6$ & $1.360(2)$ \\
$\mathrm{O} 4-\mathrm{C} 4$ & $1.209(2)$ & $\mathrm{N} 1-\mathrm{C} 2$ & $1.378(2)$ \\
$\mathrm{O} 6-\mathrm{C} 6$ & $1.2188(19)$ & $\mathrm{N} 1-\mathrm{H} 1$ & $0.89(2)$
\end{tabular}




\begin{tabular}{|c|c|c|c|}
\hline $\mathrm{O} 7-\mathrm{C} 5$ & $1.405(2)$ & $\mathrm{N} 3-\mathrm{C} 4$ & $1.368(2)$ \\
\hline $\mathrm{O} 7-\mathrm{H} 7$ & $0.87(2)$ & $\mathrm{N} 3-\mathrm{C} 2$ & $1.368(2)$ \\
\hline $\mathrm{O} 8-\mathrm{C} 5$ & $1.3706(19)$ & N3- 33 & $0.81(2)$ \\
\hline $\mathrm{O} 8-\mathrm{H} 8$ & $0.80(2)$ & $\mathrm{C} 4-\mathrm{C} 5$ & $1.536(2)$ \\
\hline $\mathrm{O} 1 \mathrm{~W}-\mathrm{H} 1 \mathrm{~W}$ & $0.92(3)$ & $\mathrm{C} 5-\mathrm{C} 6$ & $1.527(2)$ \\
\hline $\mathrm{O} 1 \mathrm{~W}-\mathrm{H} 2 \mathrm{~W}$ & $0.82(3)$ & & \\
\hline $\mathrm{C} 5-\mathrm{O} 7-\mathrm{H} 7$ & $104.6(16)$ & $\mathrm{O} 4-\mathrm{C} 4-\mathrm{N} 3$ & $123.23(15)$ \\
\hline $\mathrm{C} 5-\mathrm{O} 8-\mathrm{H} 8$ & $109.7(16)$ & $\mathrm{O} 4-\mathrm{C} 4-\mathrm{C} 5$ & $120.38(15)$ \\
\hline $\mathrm{H} 1 \mathrm{~W}-\mathrm{O} 1 \mathrm{~W}-\mathrm{H} 2 \mathrm{~W}$ & $115(3)$ & $\mathrm{N} 3-\mathrm{C} 4-\mathrm{C} 5$ & $116.18(14)$ \\
\hline $\mathrm{C} 6-\mathrm{N} 1-\mathrm{C} 2$ & $126.62(15)$ & $\mathrm{O} 8-\mathrm{C} 5-\mathrm{O} 7$ & $115.10(14)$ \\
\hline $\mathrm{C} 6-\mathrm{N} 1-\mathrm{H} 1$ & $119.2(13)$ & $\mathrm{O} 8-\mathrm{C} 5-\mathrm{C} 6$ & $106.53(13)$ \\
\hline $\mathrm{C} 2-\mathrm{N} 1-\mathrm{H} 1$ & $113.9(13)$ & $\mathrm{O} 7-\mathrm{C} 5-\mathrm{C} 6$ & $107.51(13)$ \\
\hline $\mathrm{C} 4-\mathrm{N} 3-\mathrm{C} 2$ & $125.45(14)$ & $\mathrm{O} 8-\mathrm{C} 5-\mathrm{C} 4$ & $112.19(14)$ \\
\hline $\mathrm{C} 4-\mathrm{N} 3-\mathrm{H} 3$ & $116.6(15)$ & $\mathrm{O} 7-\mathrm{C} 5-\mathrm{C} 4$ & $102.10(12)$ \\
\hline $\mathrm{C} 2-\mathrm{N} 3-\mathrm{H} 3$ & $117.1(16)$ & $\mathrm{C} 6-\mathrm{C} 5-\mathrm{C} 4$ & $113.50(13)$ \\
\hline $\mathrm{O} 2-\mathrm{C} 2-\mathrm{N} 3$ & $123.04(15)$ & $\mathrm{O} 6-\mathrm{C} 6-\mathrm{N} 1$ & $121.83(15)$ \\
\hline $\mathrm{O} 2-\mathrm{C} 2-\mathrm{N} 1$ & $119.95(15)$ & $\mathrm{O} 6-\mathrm{C} 6-\mathrm{C} 5$ & $121.52(14)$ \\
\hline $\mathrm{N} 3-\mathrm{C} 2-\mathrm{N} 1$ & $117.00(15)$ & $\mathrm{N} 1-\mathrm{C} 6-\mathrm{C} 5$ & $116.49(14)$ \\
\hline $\mathrm{C} 4-\mathrm{N} 3-\mathrm{C} 2-\mathrm{O} 2$ & $-175.55(16)$ & $\mathrm{O} 4-\mathrm{C} 4-\mathrm{C} 5-\mathrm{C} 6$ & $-159.76(15)$ \\
\hline $\mathrm{C} 4-\mathrm{N} 3-\mathrm{C} 2-\mathrm{N} 1$ & $4.2(2)$ & $\mathrm{N} 3-\mathrm{C} 4-\mathrm{C} 5-\mathrm{C} 6$ & $25.3(2)$ \\
\hline $\mathrm{C} 6-\mathrm{N} 1-\mathrm{C} 2-\mathrm{O} 2$ & $-178.33(16)$ & $\mathrm{C} 2-\mathrm{N} 1-\mathrm{C} 6-\mathrm{O} 6$ & $-177.16(16)$ \\
\hline $\mathrm{C} 6-\mathrm{N} 1-\mathrm{C} 2-\mathrm{N} 3$ & $1.9(2)$ & $\mathrm{C} 2-\mathrm{N} 1-\mathrm{C} 6-\mathrm{C} 5$ & $7.3(2)$ \\
\hline $\mathrm{C} 2-\mathrm{N} 3-\mathrm{C} 4-\mathrm{O} 4$ & $166.85(16)$ & $\mathrm{O} 8-\mathrm{C} 5-\mathrm{C} 6-\mathrm{O} 6$ & $40.3(2)$ \\
\hline $\mathrm{C} 2-\mathrm{N} 3-\mathrm{C} 4-\mathrm{C} 5$ & $-18.4(2)$ & $\mathrm{O} 7-\mathrm{C} 5-\mathrm{C} 6-\mathrm{O} 6$ & $-83.59(19)$ \\
\hline $\mathrm{O} 4-\mathrm{C} 4-\mathrm{C} 5-\mathrm{O} 8$ & $-38.9(2)$ & $\mathrm{C} 4-\mathrm{C} 5-\mathrm{C} 6-\mathrm{O} 6$ & $164.28(15)$ \\
\hline $\mathrm{N} 3-\mathrm{C} 4-\mathrm{C} 5-\mathrm{O} 8$ & $146.15(14)$ & $\mathrm{O} 8-\mathrm{C} 5-\mathrm{C} 6-\mathrm{N} 1$ & $-144.19(14)$ \\
\hline $\mathrm{O} 4-\mathrm{C} 4-\mathrm{C} 5-\mathrm{O} 7$ & $84.86(18)$ & $\mathrm{O} 7-\mathrm{C} 5-\mathrm{C} 6-\mathrm{N} 1$ & $91.92(17)$ \\
\hline $\mathrm{N} 3-\mathrm{C} 4-\mathrm{C} 5-\mathrm{O} 7$ & $-90.07(16)$ & $\mathrm{C} 4-\mathrm{C} 5-\mathrm{C} 6-\mathrm{N} 1$ & $-20.2(2)$ \\
\hline
\end{tabular}

Hydrogen-bond geometry $\left(\AA,{ }^{\circ}\right)$

\begin{tabular}{lllll}
\hline$D-\mathrm{H} \cdots A$ & $D-\mathrm{H}$ & $\mathrm{H} \cdots A$ & $D \cdots A$ & $D-\mathrm{H} \cdots A$ \\
\hline $\mathrm{N} 1-\mathrm{H} 1 \cdots \mathrm{O} 6^{\mathrm{i}}$ & $0.89(2)$ & $1.95(2)$ & $2.8366(18)$ & $170.7(19)$ \\
$\mathrm{N} 3-\mathrm{H} 3 \cdots 4^{\mathrm{ii}}$ & $0.81(2)$ & $2.11(2)$ & $2.8736(18)$ & $157(2)$ \\
$\mathrm{O} 7-\mathrm{H} 7 \cdots 2^{\mathrm{iii}}$ & $0.87(2)$ & $1.83(3)$ & $2.6958(17)$ & $173(2)$ \\
$\mathrm{O} 8-\mathrm{H} 8 \cdots \mathrm{O} 1 W^{\mathrm{iii}}$ & $0.80(2)$ & $1.87(2)$ & $2.6380(19)$ & $161(2)$ \\
$\mathrm{O} 1 W-\mathrm{H} 1 W^{\cdots} \cdots 6^{\mathrm{iv}}$ & $0.92(3)$ & $2.04(3)$ & $2.9516(19)$ & $173(2)$ \\
$\mathrm{O} 1 W-\mathrm{H} 2 W \cdots \mathrm{O} 7^{\mathrm{ii}}$ & $0.82(3)$ & $2.28(3)$ & $2.9973(19)$ & $147(3)$
\end{tabular}

Symmetry codes: (i) $-x+1,-y,-z+2$; (ii) $-x+1,-y+1,-z+1$; (iii) $x+1, y, z$; (iv) $-x+1,-y,-z+1$. 